\title{
ON THE OPENNESS AND DISCRETENESS OF MAPPINGS WITH UNBOUNDED CHARACTERISTIC OF QUASICONFORMALITY
}

\author{
E. A. Sevost'yanov
}

UDC 517.5

\begin{abstract}
The paper is devoted to the investigation of topological properties of space mappings. It is shown that orientation-preserving mappings $f: D \rightarrow \overline{\mathbb{R}^{n}}$ in a domain $D \subset \mathbb{R}^{n}, n \geq 2$, which are more general than mappings with bounded distortion, are open and discrete if a function $Q$ corresponding to the control of the distortion of families of curves under these mappings has slow growth in the domain $f(D)$, e.g., if $Q$ has finite mean oscillation at an arbitrary point $y_{0} \in f(D)$.
\end{abstract}

\section{Introduction}

For the main definitions and notation used in the present paper, see, e.g., [1]. In what follows,

$$
A\left(r_{1}, r_{2}, x_{0}\right)=\left\{x \in \mathbb{R}^{n}: r_{1}<\left|x-x_{0}\right|<r_{2}\right\} .
$$

We say that a mapping $f: D \rightarrow \mathbb{R}^{n}$ preserves orientation if the topological index $\mu(y, f, G)$ satisfies the condition $\mu(y, f, G)>0$ for an arbitrary domain $G \subset D$ such that $\bar{G} \subset D$ and an arbitrary $y \in f(G) \backslash f(\partial G)$. We say that a set $H \subset \overline{\mathbb{R}^{n}}$ is everywhere discontinuous if every connected component of it degenerates into a point. In this case, we write $\operatorname{dim} H=0$, where dim denotes the topological dimension of the set $H$ (see [2]). A mapping $f: D \rightarrow \overline{\mathbb{R}^{n}}$ is called zero-dimensional if $\operatorname{dim}\left\{f^{-1}(y)\right\}=0$ for every $y \in \overline{\mathbb{R}^{n}}$. Recall that a mapping $f: D \rightarrow \mathbb{R}^{n}$ is called a mapping with bounded distortion if the following conditions are satisfied:

(i) $f \in W_{\mathrm{loc}}^{1, n}$;

(ii) the Jacobian $J(x, f)$ of the mapping $f$ at a point $x \in D$ preserves its sign almost everywhere in $D$;

(iii) for almost all $x \in D$ and a certain constant $K<\infty$, the following relation is true:

$$
\left\|f^{\prime}(x)\right\|^{n} \leq K|J(x, f)|,
$$

where, as usual, $\left\|f^{\prime}(x)\right\|:=\sup _{h \in \mathbb{R}^{n}:|h|=1}\left|f^{\prime}(x) h\right|$ (see, e.g., [3], Chap. I, Sec. 3, or Definition 2.1 in [4], Chap. I, Sec. 2).

Extensive investigations of space mappings with bounded distortion were initiated by Reshetnyak. In particular, he proved the openness and discreteness of mappings $f$ with bounded distortion (see Theorems 6.3 and 6.4 in [3], Chap. II, Sec. 6). Let $K_{O}(f)$ denote the least constant $K$ for which relation (2) remains true. For a Institute of Applied Mathematics and Mechanics, Ukrainian National Academy of Sciences, Donetsk, Ukraine.

Translated from Ukrains'kyi Matematychnyi Zhurnal, Vol. 63, No. 8, pp. 1128-1134, August, 2011. Original article submitted December 7, 2010; revision submitted March 17, 2011. 
mapping $f: D \rightarrow \mathbb{R}^{n}$, set $E \subset D$, and $y \in \mathbb{R}^{n}$, we define the multiplicity function $N(y, f, E)$ as the number of preimages of the point $y$ in the set $E$, i.e., $N(y, f, E)=\operatorname{card}\{x \in E: f(x)=y\}$, and denote

$$
N(f, E)=\sup _{y \in \mathbb{R}^{n}} N(y, f, E)
$$

Here and in what follows, a curve $\gamma$ is understood as a continuous mapping of a segment $[a, b]$ (or an open interval $(a, b))$ into $\mathbb{R}^{n}$, i.e., $\gamma:[a, b] \rightarrow \mathbb{R}^{n}$. A family of curves $\Gamma$ is understood as a certain fixed collection of curves $\gamma$, and $f(\Gamma)=\{f \circ \gamma \mid \gamma \in \Gamma\}$. For the definitions presented below, see, e.g., [5], Chap. I, Secs. 1-6. A Borel function $\rho: \mathbb{R}^{n} \rightarrow[0, \infty]$ is called admissible for a family $\Gamma$ of curves $\gamma$ in $\mathbb{R}^{n}$ if the curvilinear integral of the first kind

$$
\int_{\gamma} \rho(x) d s
$$

satisfies the condition

$$
\int_{\gamma} \rho(x) d s \geq 1
$$

for all curves $\gamma \in \Gamma$. In this case, we write $\rho \in \operatorname{adm} \Gamma$. The modulus of a family of curves $\Gamma$ is defined as follows:

$$
M(\Gamma)=\inf _{\rho \in \operatorname{adm} \Gamma} \int_{D} \rho^{n}(x) d m(x) .
$$

To a certain extent, properties of the modulus are analogous to properties of the Lebesgue measure $m$ in $\mathbb{R}^{n}$ (see, e.g., Theorem 6.2 in [5]). In particular, for arbitrary families $\Gamma_{i}$ of curves $\gamma$ in $\mathbb{R}^{n}$, the modulus possesses the property of semiadditivity (see [5]):

$$
M\left(\bigcup_{i=1}^{\infty} \Gamma_{i}\right) \leq \sum_{i=1}^{\infty} M\left(\Gamma_{i}\right) .
$$

It is known that, for an arbitrary mapping $f: D \rightarrow \mathbb{R}^{n}$ with bounded distortion, one has

$$
M(\Gamma) \leq N(f, A) K_{O}(f) M(f(\Gamma))
$$

for an arbitrary Borel set $A$ in the domain $D$ such that $N(f, A)<\infty$ and an arbitrary family $\Gamma$ of curves $\gamma$ in $A$ (see Theorem 3.2 in [6] or Theorem 6.7 in [4], Chap. II). In the present paper, we consider mappings that, for a given Lebesgue-measurable function $Q(x), Q: \widetilde{D} \rightarrow[1, \infty]$, satisfy estimates more general than (4), namely, estimates of the form

$$
M(\Gamma) \leq \int_{\widetilde{D}} Q(y) \rho_{*}^{n}(y) d m(y),
$$


where $\rho_{*}$ is an arbitrary function such that an arbitrary curve $\gamma_{*} \in f(\Gamma)$ has at least unit length in the metric $\rho_{*}$, i.e.,

$$
\int_{\gamma_{*}} \rho_{*}(y) d s \geq 1 \quad \forall \gamma_{*} \in f(\Gamma),
$$

and $\widetilde{D} \subset f(D)$. The function $Q(y)$ in (5), is, generally speaking, unbounded [see, e.g., inequality (8.5) in [7], Chap. VIII]. Relations of the form (5) were established for many classes of mappings, e.g., for so-called mappings with finite distortion of length for specific values of $Q(y)$ (see, e.g., Theorem 8.5 in [7]; see also [8]). Note that if $Q(y) \leq K$ almost everywhere, then, under the additional condition of homeomorphism of the mapping $f$, inequality (5) defines quasiconformal mappings, and only them (see Definition 13.1 and Theorem 34.3 in [5]). Also note that, even in the case of a bounded function $Q(y)$ in relation (5), the corresponding mapping $f$, which, a priori, is not orientation-preserving, must be neither open, nor discrete, nor homeomorphic (see, e.g., Sec. 8.10 in [7]).

It is clear that if the multiplicity function $N(f, A)$ in (4) is finite, then, by definition, the orientation-preserving mapping $f$ is discrete, and, hence, by virtue of the corollary in [9, p. 333], it is open. We pose the following inverse problem: If an inequality of the form (5) is true, then what can be said about the discreteness and openness of the mapping $f$ ?

We now introduce some additional definitions and notation. Let $Q: D \rightarrow[0, \infty]$ be a Lebesgue-measurable function. Then $q_{x_{0}}(r)$ denotes the integral mean value of $Q(x)$ over the sphere $S\left(x_{0}, r\right)$, namely,

$$
q_{x_{0}}(r):=\frac{1}{\omega_{n-1} r^{n-1}} \int_{\left|x-x_{0}\right|=r} Q(x) d S,
$$

where $d S$ is an element of the area of the surface $S$. We say that a function $\varphi: D \rightarrow \mathbb{R}$ has finite mean oscillation at a point $x_{0} \in D \quad\left(\varphi \in \mathrm{FMO}\left(x_{0}\right)\right)$ if (see, e.g., [7], Sec. 6.1, Chap. VI)

$$
\limsup _{\varepsilon \rightarrow 0} \frac{1}{\Omega_{n} \varepsilon^{n}} \int_{B\left(x_{0}, \varepsilon\right)}\left|\varphi(x)-\bar{\varphi}_{\varepsilon}\right| d m(x)<\infty,
$$

where

$$
\bar{\varphi}_{\varepsilon}=\frac{1}{\Omega_{n} \varepsilon^{n}} \int_{B\left(x_{0}, \varepsilon\right)} \varphi(x) d m(x) .
$$

The main result of the present paper is the following theorem:

Theorem 1. Let $f: D \rightarrow \mathbb{R}^{n}$ be an orientation-preserving mapping. Suppose that, for every domain $D^{\prime} \subset$ $f(D), \overline{D^{\prime}} \subset f(D)$, there exists a function $Q: D^{\prime} \rightarrow[1, \infty]$ such that, for an arbitrary family $\Gamma$ of curves $\gamma$ in $D$ and an arbitrary function $\rho_{*}(y) \in \operatorname{adm} f(\Gamma)$, a relation of the form (5) is true. Suppose that the function $Q(y)$ satisfies at least one of the following conditions:

(i) $Q$ belongs to $\operatorname{FMO}\left(y_{0}\right)$ at an arbitrary point $y_{0} \in D^{\prime}$;

(ii) $q_{y_{0}}(r)=O\left(\left[\log \frac{1}{r}\right]^{n-1}\right)$ as $r \rightarrow 0$ for all $y_{0} \in D^{\prime}$, where the function $q_{y_{0}}(r)$ is defined by (6); 
(iii) for every $y_{0} \in D^{\prime}$, there exists a number $\delta\left(y_{0}\right)>0, \delta\left(y_{0}\right)<\operatorname{dist}\left(y_{0}, \partial D^{\prime}\right)$, such that

$$
\int_{0}^{\delta\left(y_{0}\right)} \frac{d t}{t q_{y_{0}}^{\frac{1}{n-1}}(t)}=\infty
$$

Then the mapping $f$ is open and discrete.

Remark 1. Strictly speaking, the function $Q$ in Theorem 1 depends on the domain $D^{\prime}$ and should have been denoted by $Q_{D^{\prime}}$. Nevertheless, we omit this subscript, lest we complicate the notation.

Note that if $Q(x) \equiv K=$ const, then Theorem 1 establishes the openness and discreteness of mappings that satisfy the condition $M(\Gamma) \leq K M(f(\Gamma))$, where, generally speaking, the constant $K=K_{D^{\prime}}$ depends on the domain $D^{\prime}$. In particular, all mappings with bounded distortion satisfy inequalities of this type in the case where $Q(x)=N\left(f, D^{\prime}\right) K_{O}(f)$ [see relation (4)].

Theorem 1 remains true for mappings of the form $f: D \rightarrow \overline{\mathbb{R}^{n}}$ under the condition that assertions (i)-(iii) are reformulated at the point $y_{0}=0$ for the mapping $\tilde{f}=f \circ \varphi$, where

$$
\varphi(x)=\frac{x}{|x|^{2}}, \quad \varphi: \infty \mapsto 0
$$

\section{Formulation and Proof of the Main Lemma}

We call a connected compact set $C \subset \overline{\mathbb{R}^{n}}$ a continuum. Let $E, F \subset \overline{\mathbb{R}^{n}}$ be arbitrary sets. Let $\Gamma(E, F, D)$ denote the family of all curves $\gamma:[a, b] \rightarrow \overline{\mathbb{R}^{n}}$ that connect $E$ and $F$ in $D$, i.e., $\gamma(a) \in E, \gamma(b) \in F$, and $\gamma(t) \in D$ for $t \in(a, b)$. We say that a family of curves $\Gamma_{1}$ is minorized by a family $\Gamma_{2}\left(\Gamma_{1}>\Gamma_{2}\right)$ if, for every curve $\gamma \in \Gamma_{1}$, there exists a subcurve that belongs to the family $\Gamma_{2}$. In this case, we have $M\left(\Gamma_{1}\right) \leq M\left(\Gamma_{2}\right)$ (see, e.g., Theorem 6.4 in [5]). The following lemma contains the main result of the present paper in the most general case:

Lemma 1. Let $f: D \rightarrow \mathbb{R}^{n}$ be an orientation-preserving mapping. Suppose that, for every domain $G \subset$ $f(D)$ such that $\bar{G} \subset f(D)$, there exists a Lebesgue-measurable function $Q: G \rightarrow[1, \infty]$ such that

$$
M(\Gamma) \leq \int_{f(D)} Q(y) \rho_{*}^{n}(y) d m(y)
$$

for an arbitrary family $\Gamma$ of curves $\gamma$ in $G$ and an arbitrary function $\rho_{*}(y) \in \operatorname{adm} f(\Gamma)$. Further, suppose that, for every $y_{0} \in G$, there exists $\varepsilon\left(y_{0}\right)>0$ such that

$$
\int_{A\left(\varepsilon, \varepsilon\left(y_{0}\right), y_{0}\right)} Q(y) \psi^{n}\left(\left|y-y_{0}\right|\right) d m(y)=o\left(I^{n}\left(\varepsilon, \varepsilon_{0}\right)\right)
$$

for a certain Borel function $\psi(t):(0, \infty) \rightarrow[0, \infty]$ such that

$$
0<I\left(\varepsilon, \varepsilon\left(y_{0}\right)\right):=\int_{\varepsilon}^{\varepsilon\left(y_{0}\right)} \psi(t) d t<\infty
$$


for all $\varepsilon \in\left(0, \varepsilon\left(y_{0}\right)\right)$, where $A\left(\varepsilon, \varepsilon\left(y_{0}\right), y_{0}\right)$ is defined by $(1)$ for $r_{1}=\varepsilon, r_{2}=\varepsilon\left(y_{0}\right)$, and $x_{0}=y_{0}$. Then the mapping $f$ is open and discrete.

Remark 2. Under the conditions of Lemma 1, we can assume that, for an arbitrary fixed $A$ such that $0<$ $A<\varepsilon\left(y_{0}\right)$ and all $\varepsilon \in(0, A)$, a condition of the form

$$
\int_{\varepsilon}^{A} \psi(t) d t>0
$$

is satisfied. Indeed, it follows from (9) and (10) that

$$
\int_{\varepsilon}^{A} \psi(t) d t \rightarrow \infty \quad \text { as } \quad \varepsilon \rightarrow 0
$$

because $Q \geq 1$ and the value of the integral on the left-hand side of (9) increases as $\varepsilon$ decreases.

Proof of Lemma 1. Since every orientation-preserving mapping $f: D \rightarrow \mathbb{R}^{n}$ is open and discrete in the domain $D$ (see, e.g., the corollary in [9, p.333]), to prove the lemma it suffices to show that $f$ is a zero-dimensional mapping. Assume the contrary. Then there exists $y_{0} \in \mathbb{R}^{n}$ such that the $\operatorname{set}\left\{f^{-1}\left(y_{0}\right)\right\}$ is not everywhere discontinuous. Therefore, by definition, there exists a continuum $C \subset\left\{f^{-1}\left(y_{0}\right)\right\}$. Since the mapping $f$ preserves orientation, we have $f \not \equiv y_{0}$. According to the theorem on the preservation of a sign, there exist $x_{0} \in D$ and $\varepsilon_{0}>0$ such that $\overline{B\left(x_{0}, \varepsilon_{0}\right)} \subset D$ and

$$
f(x) \neq y_{0} \quad \forall x \in \overline{B\left(x_{0}, \varepsilon_{0}\right)}
$$

We arbitrarily choose a domain $G_{1} \subset D$ such that $\overline{G_{1}} \subset D$ so that $C \cup \overline{B\left(x_{0}, \varepsilon_{0}\right)} \subset G_{1}$. Then, by virtue of Lemma 1.15 in [10], we get

$$
M\left(\Gamma\left(C, \overline{B\left(x_{0}, \varepsilon_{0}\right)}, G_{1}\right)\right)>0
$$

Note that, by virtue of inequality (11) and the relation $f(C)=\left\{y_{0}\right\}$, none of the curves of the family $\Delta=$ $f\left(\Gamma\left(C, \overline{B\left(x_{0}, \varepsilon_{0}\right)}, G_{1}\right)\right)$ degenerates into a point. At the same time, all curves of the family $\Delta$ have the point $y_{0}$ as their endpoint. Let $\Gamma_{i}$ be a family of curves $\alpha_{i}(t):(0,1) \rightarrow \mathbb{R}^{n}$ such that $\alpha_{i}(1) \in S\left(y_{0}, r_{i}\right), r_{i}<\varepsilon\left(y_{0}\right)$, where $r_{i}$ is a certain strictly positive real sequence such that $r_{i} \rightarrow 0$ as $i \rightarrow \infty$ and $\alpha_{i}(t) \rightarrow y_{0}$ as $t \rightarrow 0$. Then

$$
\Gamma\left(C, \overline{B\left(x_{0}, \varepsilon_{0}\right)}, G_{1}\right)=\bigcup_{i=1}^{\infty} \Gamma_{i}^{*}
$$

where $\Gamma_{i}^{*}$ is the subfamily of all curves $\gamma$ from $\Gamma\left(C, \overline{B\left(x_{0}, \varepsilon_{0}\right)}, G_{1}\right)$ such that $f(\gamma)$ has a subcurve in $\Gamma_{i}$. We fix $i \in \mathbb{N}$ and, for every $\varepsilon \in\left(0, r_{i}\right)$, consider the family of all curves $\Gamma_{i, \varepsilon}$ that connect the spheres $S\left(y_{0}, r_{i}\right)$ and $S\left(y_{0}, \varepsilon\right)$ in $f\left(G_{1}\right)$. Note that, for an any $\varepsilon \in\left(0, r_{i}\right)$, we have

$$
\Gamma_{i}>\Gamma_{i, \varepsilon}
$$


Consider the function

$$
\rho_{i, \varepsilon}(y)= \begin{cases}\psi\left(\left|y-y_{0}\right|\right) / I\left(\varepsilon, r_{i}\right), & y \in A\left(\varepsilon, r_{i}, y_{0}\right), \\ 0, & y \in \overline{\mathbb{R}^{n}} \backslash A\left(\varepsilon, r_{i}, y_{0}\right),\end{cases}
$$

where

$$
I\left(\varepsilon, r_{i}\right)=\int_{\varepsilon}^{r_{i}} \psi(t) d t
$$

Note that $\rho_{i, \varepsilon}(y) \in \operatorname{adm} \Gamma_{i, \varepsilon}$. Indeed, by virtue of Theorem 5.7 in [5], the integral of an arbitrary radial function $\Psi\left(\left|y-y_{0}\right|\right)$ along a curve that connects the spheres $S\left(y_{0}, r_{i}\right)$ and $S\left(y_{0}, \varepsilon\right)$ is not less than the corresponding integral of the function $\Psi(t)$ over the segment $\left(\varepsilon, r_{i}\right)$, namely,

$$
\int_{\gamma} \rho_{i, \varepsilon}(y) d s \geq \frac{1}{I\left(\varepsilon, r_{i}\right)} \int_{\varepsilon}^{r_{i}} \psi(t) d t=1
$$

for any curve $\gamma \in \Gamma_{i, \varepsilon}$. Therefore, according to (14), we have $\rho_{i, \varepsilon}(y) \in \operatorname{adm} \Gamma_{i}$ and, by virtue of relation (8),

$$
M\left(\Gamma_{i}^{*}\right) \leq \int_{f(D)} Q(y) \rho_{i, \varepsilon}^{n}(y) d m(y)=\int_{A\left(\varepsilon, \varepsilon\left(y_{0}\right), y_{0}\right)} Q(y) \rho_{i, \varepsilon}^{n}(y) d m(y) \leq \mathfrak{F}_{i}(\varepsilon)
$$

where

$$
\mathfrak{F}_{i}(\varepsilon)=\frac{1}{I\left(\varepsilon, r_{i}\right)^{n}} \int_{A\left(\varepsilon, \varepsilon\left(y_{0}\right), y_{0}\right)} Q(y) \psi^{n}\left(\left|y-y_{0}\right|\right) d m(y)
$$

and

$$
I\left(\varepsilon, r_{i}\right)=\int_{\varepsilon}^{r_{i}} \psi(t) d t
$$

Taking (9) into account, we obtain

$$
\int_{A\left(\varepsilon, \varepsilon\left(y_{0}\right), y_{0}\right)} Q(y) \psi^{n}\left(\left|y-y_{0}\right|\right) d m(y)=G(\varepsilon)\left(\int_{\varepsilon}^{\varepsilon\left(y_{0}\right)} \psi(t) d t\right)^{n},
$$

where $G(\varepsilon) \rightarrow 0$ as $\varepsilon \rightarrow 0$ by virtue of the conditions of the lemma. Note that

$$
\mathfrak{F}_{i}(\varepsilon)=G(\varepsilon)\left(1+\frac{\int_{r_{i}}^{\varepsilon\left(y_{0}\right)} \psi(t) d t}{\int_{\varepsilon}^{r_{i}} \psi(t) d t}\right)^{n},
$$


where

$$
\int_{r_{i}}^{\varepsilon\left(y_{0}\right)} \psi(t) d t<\infty
$$

is a fixed number and

$$
\int_{\varepsilon}^{r_{i}} \psi(t) d t \rightarrow \infty \quad \text { as } \quad \varepsilon \rightarrow 0
$$

because the value of the integral on the left-hand side of (9) increases as $\varepsilon$ decreases. Thus, $\mathfrak{F}_{i}(\varepsilon) \rightarrow 0$ as $\varepsilon \rightarrow 0$. Passing to the limit as $\varepsilon \rightarrow 0$ in inequality (15), whose left-hand side is independent of $\varepsilon$, we obtain $M\left(\Gamma_{i}^{*}\right)=0$ for any natural $i$. However, in this case, $M\left(\Gamma\left(C, \overline{B\left(x_{0}, \varepsilon_{0}\right)}, G_{1}\right)\right)=0$ by virtue of (13) and (3), which contradicts inequality (12). The contradiction obtained proves that the mapping $f$ is zero-dimensional, and, hence, according to the corollary in [9, p.333], the mapping $f$ is open and discrete, which was to be proved.

The lemma is proved.

\section{On the Proof of the Main Result}

The statement of Theorem 1 follows directly from Lemma 1 and Lemma 8 in [1].

Remark 3. The condition $Q(x) \geq 1$ guarantees that $q_{x_{0}}(r) \geq 1$ for almost all values of $r$. Therefore,

$$
\int_{\varepsilon_{1}}^{\varepsilon_{2}} \frac{d t}{t q_{x_{0}}^{\frac{1}{n-1}}(t)} \leq \log \frac{\varepsilon_{2}}{\varepsilon_{1}}<\infty \text { for all } \varepsilon_{1}, \varepsilon_{2}>0
$$

in this connection, see also Lemma 8 in [1].

\section{Examples}

Example 1. The most important example of mappings that satisfy estimates of the form (5) is that of so-called mappings with finite distortion of length (see, e.g., Chap. VIII in [7]). The introduction and investigation of this class are motivated by the necessity of the description of "minimum" requirements on mappings under which the distortion of the modulus of families of curves satisfies certain estimates. We introduce some notation.

The external dilation of a mapping $f$ at a point $x$ is defined as follows:

$$
K_{O}(x, f)=\frac{\left\|f^{\prime}(x)\right\|^{n}}{|J(x, f)|} \quad \text { if } \quad J(x, f) \neq 0
$$

$K_{O}(x, f)=1$ if $f^{\prime}(x)=0$, and $K_{O}(x, f)=\infty$ at the other points. We set

$$
K_{I}\left(y, f^{-1}, E\right):=\sum_{x \in E \cap f^{-1}(y)} K_{O}(x, f)
$$


Corollary 1. Let $f: D \rightarrow \mathbb{R}^{n}$ be an orientation-preserving mapping with finite distortion of length. Suppose that, for every domain $G \subset D, \bar{G} \subset D$, the function $K_{I}\left(y, f^{-1}, G\right)$ satisfies at least one of conditions (i)-(iii) of Theorem 1 at an arbitrary point $y_{0} \in f(G)$. Then the mapping $f$ is open and discrete.

Proof. Note that every mapping $f: D \rightarrow \mathbb{R}^{n}$ with finite distortion of length satisfies the inequality

$$
M(\Gamma) \leq \int_{f(E)} K_{I}\left(y, f^{-1}, E\right) \rho_{*}^{n}(y) d m(y)
$$

for any measurable set $E \subset D$, an arbitrary family $\Gamma \subset E$ of curves $\gamma$ in $E$, and every function $\rho_{*}(y) \in$ $\operatorname{adm} f(\Gamma)$ (see, e.g., Theorem 8.5 in [7], Chap. VIII). The other assertions follow from the theorem.

The corollary is proved.

Example 2. Generally speaking, the condition of preservation of orientation of the mapping $f$ in all statements presented above cannot be omitted. An example of a mapping $f$ with finite distortion of length that does not preserve orientation and is such that $M(f(\Gamma))=M(\Gamma)$, i.e., $Q \equiv 1$ in inequality (5), but is neither discrete nor open is given in Sec. 8.10, Chap. VIII in [7].

We also give another example. Let $x=\left(x_{1}, \ldots, x_{n}\right)$. We define $f$ as the identical mapping in the closed domain $\left\{x_{n} \geq 0\right\}$ and set $f(x)=\left(x_{1}, \ldots,-x_{n}\right)$ for $x_{n}<0$. This mapping is the reflection in the hyperplane $x_{n}=0$ for $x_{n}<0$ (for nonnegative values of $x_{n}$, it is the identical mapping). Note that $f$ is a mapping with finite distortion of length, and, moreover, the mapping $f$ preserves the lengths of curves. Therefore, $f$ satisfies inequality (5) for $Q \equiv 1$. This mapping is discrete but not open. For example, under the mapping $f$ the ball $\mathbb{B}^{n}$ is mapped into the semisphere $\left\{y=\left(y_{1}, \ldots, y_{n}\right) \in \mathbb{R}^{n}:|y|<1, y_{n} \geq 0\right\}$, which is not an open set in $\mathbb{R}^{n}$.

\section{REFERENCES}

1. E. A. Sevost'yanov, "On branching points of mappings with unbounded characteristic of quasiconformality," Sib. Mat. Zh., 51, No. 5, 1129-1146 (2010).

2. W. Hurewicz and H. Wallman, Dimension Theory, Princeton University, Princeton (1948).

3. Yu. G. Reshetnyak, Space Mappings with Bounded Distortion [in Russian], Nauka, Novosibirsk (1982).

4. S. Rickman, Quasiregular Mappings, Springer, Berlin (1993).

5. J. Väisälä, Lectures on n-Dimensional Quasiconformal Mappings, Springer, Berlin (1971).

6. O. Martio, S. Rickman, and J. Väisälä, "Definitions for quasiregular mappings," Ann. Acad. Sci. Fenn., Ser. A1. Math., 448, 1-40 (1969).

7. O. Martio, V. Ryazanov, U. Srebro, and E. Yakubov, Moduli in Modern Mapping Theory, Springer, New York (2009).

8. C. J. Bishop, V. Ya. Gutlyanskii, O. Martio, and M. Vuorinen, "On conformal dilatation in space," Int. J. Math. Math. Sci., 22, 1397-1420 (2003).

9. C. J. Titus and G. S. Young, “The extension of interiority with some applications," Trans. Amer. Math. Soc., 103, 329-340 (1962).

10. R. Näkki, "Boundary behavior of quasiconformal mappings in $n$-space," Ann. Acad. Sci. Fenn., Ser. A1. Math., 484, 1-50 (1970). 